\title{
SYMMETRY-BREAKINGS FOR SEMILINEAR ELLIPTIC EQUATIONS ON FINITE CYLINDRICAL DOMAINS
}

\author{
SONG-SUN LIN
}

(Communicated by Barbara L. Keyfitz)

\begin{abstract}
We study the existence and multiplicity of asymmetric positive solutions of a semilinear elliptic equation on finite cylinders with mixed type boundary conditions. By using a Nehari-type variational method, we prove that the numbers of asymmetric positive solutions are increasing without bound when the lengths of cylinders are increasing. On the contrary, by using the blow up technique, we obtain an a priori bound for positive solutions and then prove that all positive solutions must be symmetric when the cylinders are short enough.
\end{abstract}

\section{INTRODUCTION}

Let $\omega$ be a bounded smooth domain in $\mathbb{R}^{n}, n \geq 1, a>0$, and $\Omega_{a}=$ $(-a, a) \times \omega$ be a finite cylindrical domain in $\mathbb{R}^{n+1}=\left\{(x, y): x \in \mathbb{R}^{1}\right.$ and $\left.y \in \mathbb{R}^{n}\right\}$. In this paper, we shall study the existence and multiplicity of positive solutions of the following semilinear elliptic equation with mixed type boundary conditions

$$
\begin{aligned}
\Delta u+f(u)=0 & & \text { in } \Omega_{a}, \\
u=0 & & \text { on }[-a, a] \times \partial \omega, \\
\frac{\partial u}{\partial x}=0 & & \text { on }\{-a, a\} \times \omega,
\end{aligned}
$$

where $f$ satisfies the following conditions:

(H-0) $f \in C^{1}\left(\mathbb{R}^{1}\right), f(u)>0$ for $u>0$,

(H-1) $f(0)=0$ and $f^{\prime}(0)=0$,

(H-2) there exists $\sigma>0$ such that $u f^{\prime}(u) \geq(1+\sigma) f(u)$ for all $u \geq 0$,

(H-3) $f(u) \leq C\left(u^{p}+1\right)$ for some $C>0$ and all $u \geq 0$, where $1<p<$ $(n+3) /(n-1)$ if $n \geq 2$ and $1<p<\infty$ if $n=1$.

A solution $u \in C^{2}\left(\bar{\Omega}_{a}\right)$ of (1.1)-(1.3) is said to be symmetric (with respect to the $x$-axis) if $u$ is constant along the $x$-axis, i.e., $u=u(y)$ and satisfies

$$
\begin{aligned}
\Delta u+f(u)=0 & \text { in } \omega, \\
u=0 & \text { on } \partial \omega .
\end{aligned}
$$

Received by the editors January 30, 1991 and, in revised form, July 5, 1991.

1991 Mathematics Subject Classification. Primary 35B32, 35J65, 35P30.

Key words and phrases. Symmetry breaking, cylinders.

Work partially supported by the National Science Council of the Republic of China. 
Otherwise, $u$ is called an asymmetric solution. The following problem was posed by Berestycki:

For $f(u)=u^{p}$, taking the length $a$ as a bifurcation parameter, is there symmetry-breaking for $(1.1)-(1.3)$ ?

Previously, it has been known that there is an asymmetric solution when $a$ is large enough. In this paper, we answer the problem (P) partially, and hope it will lead to more complete results of the problem. The main results are as follows.

Theorem 1.1. Assume conditions $(\mathrm{H}-0)-(\mathrm{H}-3)$ are satisfied. Then there exists an increasing sequence $a_{k}=a_{k}(\omega, \sigma) \rightarrow \infty$ as $k \rightarrow \infty$, such that for each $k \geq 1$ and $a \in\left(a_{k}, \infty\right)$, (1.1)-(1.3) have at least $k$-many asymmetric positive solutions.

On the contrary, for short cylinders we have

Theorem 1.2. Assume $f$ satisfies $(\mathrm{H}-0)-(\mathrm{H}-2)$ and

$(\mathrm{H}-3)^{\prime} \lim _{u \rightarrow \infty} f(u) / u^{p}=C>0$ for $1<p<(n+3) /(n-1)$ if $n \geq 2$ and $1<p<\infty$ if $n=1$. Then there exists $a_{*}=a_{*}(\omega, p)>0$ such that for any $a \in\left(0, a_{*}\right)$, every positive solution of (1.1)-(1.3) is symmetric.

The last two theorems indicate that there are asymmetric bifurcations at certain critical numbers $a_{k}^{*}, a_{k}^{*} \rightarrow \infty$ as $k \rightarrow \infty$, and the bifurcation branches will go to the direction of increasing $a$. However, we still need more rigorous justifications.

To prove Theorem 1.1, we need to study the problems of asymmetric instability of symmetric positive solutions and then use a Nehari-type variational method to prove that there exist asymmetric positive solutions when all symmetric positive solutions are unstable with respect to certain asymmetric mode.

To prove Theorem 1.2, we need to obtain some a priori bounds for positive solutions of $(1.1)-(1.3)$ and then prove that asymmetric positive solutions cannot exist when the cylinders are short enough.

As for the related problems, the problems of symmetry-breaking of positive radial solutions on a ball were studied in [4, 6, 15-17], on annular domains in $[3,5,8-11,18,19]$, and on sectorial domains in $[2,12]$.

The paper is organized as follows. In $\S 2$ we study the linear eigenvalue problems and obtain some asymmetric instability results for symmetric positive solutions for long cylinders. In $\S 3$ we prove the existence of asymmetric positive solutions for long cylinders. In $\S 4$ we prove the nonexistence of asymmetric positive solutions for short cylinders.

\section{LINEARIZED EIGENVALUE PROBLEMS}

Let $g \in C(\bar{\omega})$. Consider the following linear eigenvalue problem:

$$
\begin{aligned}
\Delta v+g(y) v & =-\nu v & & \text { in } \omega, \\
v & =0 & & \text { on } \partial \omega .
\end{aligned}
$$

Then there is the sequence $\left\{\nu_{1}, \nu_{2}, \ldots\right\}=\left\{\nu_{1}(g), \nu_{2}(g), \ldots\right\}$ of eigenvalues of (2.1) and (2.2) satisfying $\nu_{1}<\nu_{2} \leq \nu_{3} \leq \cdots$, where each distinct eigenvalue 
is repeated according to its multiplicity. By separation of variables, the linear eigenvalue problem

$$
\begin{aligned}
\Delta w+g(y) w & =-\lambda w & & \text { in } \Omega_{a}, \\
w & =0 & & \text { on }[-a, a] \times \partial \omega, \\
\frac{\partial w}{\partial x} & =0 & & \text { on }\{-a, a\} \times \omega,
\end{aligned}
$$

is equivalent to the problems

$$
\begin{array}{cl}
\Delta v+g(y) v=-(\lambda+\mu) v & \text { in } \omega, \\
v=0 & \text { on } \partial \omega,
\end{array}
$$

and

$$
\begin{gathered}
\varphi^{\prime \prime}(x)=\mu \varphi(x), \quad x \in(-a, a), \\
\varphi^{\prime}(-a)=0=\varphi^{\prime}(a),
\end{gathered}
$$

with $w(x, y)=\varphi(x) v(y)$. It is clear that the eigenvalues of (2.8) and (2.9) are given by

$$
\mu_{k}(a)=-(k \pi / 2 a)^{2},
$$

$k=0,1,2, \ldots$, and the associated eigenfunctions are

$$
\varphi_{k}(x)= \begin{cases}\sin \frac{k \pi}{2 a} x & \text { if } k \text { is odd } \\ \cos \frac{k \pi}{2 a} x & \text { if } k \text { is even }\end{cases}
$$

Therefore, $\lambda$ is an eigenvalue of (2.3)-(2.5) if and only if $\lambda+\mu_{k}=\nu_{l}$ for some $k$ and $l$. Hence, we denote

$$
\lambda_{k, l}=\lambda_{k, l}(g, a)=\nu_{l}-\mu_{k}=\nu_{l}(g)+\left(\frac{k \pi}{2 a}\right)^{2}
$$

where $k=0,1,2, \ldots$ and $l=1,2, \ldots$. Note that when $k=0$ it corresponds to symmetric modes and $k \geq 1$ to asymmetric modes.

Let $u_{0}$ be any symmetric positive solution and $\nu_{1}=\nu_{1}\left(u_{0}\right)$ be the least eigenvalue of (2.1) and (2.2) with $g(y)=f^{\prime}\left(u_{0}(y)\right)$. Then we have the following estimate for $\nu_{1}\left(u_{0}\right)$.

Lemma 2.1. Assume condition (H-2) is satisfied. Then for any symmetric positive solution $u_{0}$ we have

$$
\nu_{1}\left(u_{0}\right) \leq-\sigma \bar{\nu}_{1},
$$

where $\bar{\nu}_{1}=\bar{\nu}_{1}(\omega)>0$ is the least eigenvalue of the Laplacian $-\Delta$ on $\omega$ with Dirichlet boundary conditions.

Proof. It is well known that $\nu_{1}\left(u_{0}\right)$ can be characterized by

$$
\nu_{1}=\inf \left\{Q(v) / \int_{\omega} v^{2}: v \in H_{0}^{1}(\omega) \backslash\{0\}\right\},
$$

where

$$
Q(v)=\int_{\omega}|\nabla v|^{2}-f^{\prime}\left(u_{0}\right) v^{2}
$$


Since $u_{0}$ is a solution of $(1.4)$ and (1.5), we have

$$
\int_{\omega}\left|\nabla u_{0}\right|^{2}=\int_{\omega} u_{0} f\left(u_{0}\right)
$$

Hence, by (H-2), we have

$$
\begin{aligned}
Q\left(u_{0}\right) & =\int_{\omega}\left|\nabla u_{0}\right|^{2}-f^{\prime}\left(u_{0}\right) u_{0}^{2}=\int_{\omega} u_{0} f\left(u_{0}\right)-f^{\prime}\left(u_{0}\right) u_{0}^{2} \\
& \leq-\sigma \int_{\omega} u_{0} f\left(u_{0}\right)=-\sigma \int_{\omega}\left|\nabla u_{0}\right|^{2} .
\end{aligned}
$$

Therefore, by using the Poincaré inequality, (2.13) follows. The proof is complete.

Note that the upper bound $-\sigma \bar{\nu}_{1}$ of $\nu_{1}\left(u_{0}\right)$ is independent of symmetric positive solutions $u_{0}$.

A consequence of Lemma 2.1 is the following asymmetric instability results for long cylinders.

Theorem 2.2. Assume condition (H-2) is satisfied. For each $k \geq 1$, let

$$
a_{k}=\frac{k \pi}{2}\left(\sigma \bar{\nu}_{1}\right)^{-1 / 2}
$$

Then for any $a \in\left(a_{k}, \infty\right)$ and any symmetric positive solution $u_{0}$ we have $\lambda_{k, 1}\left(u_{0}, a\right)<0$.

Proof. By (2.12), $\lambda_{k, 1}\left(u_{0}, a\right)=\nu_{1}\left(u_{0}\right)+\mu_{k}(a)$; the result follows from Lemma 2.1.

Remark 2.3. Assume conditions $(\mathrm{H}-0)-(\mathrm{H}-3)$ are satisfied.

(i) If $u \in C^{2}\left(\Omega_{a}\right) \cap C^{0}\left(\bar{\Omega}_{a}\right)$ is a solution of $(1.1)-(1.3)$, then $u$ is positive in $\Omega$. Indeed, by $(\mathrm{H}-0)$ and the maximum principle, the minimum of $u$ in $\bar{\Omega}_{a}$ is achieved on $\partial \Omega_{a}$. On the other hand, by (1.3) and the strong maximum principle, the minimum cannot be achieved on $\{-a, a\} \times \omega$. Hence the minimum will be achieved on $[-a, a] \times \partial \omega$, which implies $u$ is positive in $\Omega_{a}$.

(ii) There exist positive solutions of (1.1)-(1.3) and (1.4) and (1.5), respectively, see, e.g., $[1,14]$.

\section{LONG CYLINDERS}

In this section, we shall prove that if the cylinders are long enough, $a>a_{k}$, then there exist at least $k$-many asymmetric positive solutions. We shall use a Nehari-type variational method.

For any function $u$ defined on $\Omega_{a}$, it can be extended to $\tilde{u}$ on $\Omega_{\infty}=$ $(-\infty, \infty) \times \omega$. In fact, we first define

$$
\tilde{u}(x, y)=u(2 a-x, y) \text { for } x \in(a, 3 a) \text { and } y \in \omega,
$$

a reflection with respect to $x=a$. Then $\tilde{u}$ is extended to the whole of $\Omega_{\infty}$ by $4 a$ periodically along $x$-axis. Due to the Neumann boundary conditions on $\{-a, a\} \times \omega$, the eigenfunctions $w_{k, l}$ and solutions of (1.1)-(1.3) can be extended smoothly on $\Omega_{\infty}$ in this way. 
We need some notation. Let $V=\left\{u \in H^{1}\left(\Omega_{a}\right): u=0\right.$ on $\left.[-a, a] \times \partial \omega\right\}$. Define the functionals

$$
J(u)=\int_{\Omega_{a}} \frac{1}{2}|\nabla u|^{2}-F(u) \text { and } M(u)=\int_{\Omega_{a}}|\nabla u|^{2}-u f(u)
$$

on $V$, where $F(u)=\int_{0}^{u} f(t) d t$.

For each $k \geq 1$, the submanifolds $V_{k}$ and $V_{\infty}$ of $V$ are defined by

$$
\begin{aligned}
V_{k}=V_{k, a}=\left\{u \in V: \tilde{u}\left(x+\frac{4 a}{k}, y\right)=\tilde{u}(x, y)\right. \\
\left.\qquad \text { for }(x, y) \in \Omega_{\infty} \text { and } M(u)=0\right\}
\end{aligned}
$$

and

$$
V_{\infty}=\left\{u \in H_{0}^{1}(\omega): M(u)=0\right\}
$$

Let

$$
I_{k}=I_{k}(a)=\inf \left\{J(u): u \in V_{k}\right\}, \quad I_{\infty}=I_{\infty}(a)=\inf \left\{J(u): u \in V_{\infty}\right\} .
$$

Let $u_{0}$ be a symmetric positive solution, and $w_{0}=v_{1}(y)>0$ and $w_{k}=$ $\varphi_{k}(x) v_{1}(y)$ be eigenfunctions with respect to eigenvalues $\lambda_{0,1}=\lambda_{0,1}\left(u_{0}, a\right)$ and $\lambda_{k, 1}=\lambda_{k, 1}\left(u_{0}, a\right)$ with $\int_{\Omega_{a}} w_{0}^{2}=\int_{\Omega_{a}} w_{k}^{2}=1$. Note that for $k \geq 1$, we have

$$
\int_{\Omega_{a}} w_{k}=0
$$

Then we have the following results concerning the change of $J(u)$ along the direction of asymmetric mode $w_{k}$.

Lemma 3.1. Assume conditions $(\mathrm{H}-0)-(\mathrm{H}-2)$ are satisfied. Then there exist $\varepsilon>0$ and a smooth function $\delta:(-\varepsilon, \varepsilon) \rightarrow \mathbb{R}^{1}$ with $\delta(0)=\delta^{\prime}(0)=0$ such that

$$
M\left(u_{0}+\delta(t) w_{0}+t w_{k}\right)=0
$$

for all $t \in(-\varepsilon, \varepsilon)$. Furthermore, we have

$$
J\left(u_{0}+\delta(t) w_{0}+t w_{k}\right)=J\left(u_{0}\right)+\frac{1}{2} \lambda_{0,1} \delta(t)^{2}+\frac{1}{2} \lambda_{k, 1} t^{2}+O\left(t^{4}\right),
$$

as $t \sim 0$.

Proof. The proofs are similar to Lemmas 6.1 and 6.2 in [11]. (3.2) is proved by using the Implicit Function Theorem and (3.3) is proved by straightforward computations; the details are omitted. Note that $(3.1)$ is used repeatedly in the proofs.

To prove Theorem 1.1, we need the following result, which indicates that asymmetric minimizers are different for different modes.

Lemma 3.2. Assume conditions $(\mathrm{H}-0)-(\mathrm{H}-3)$ are satisfied. If $I_{k m}<I_{\infty}$ then $I_{k}<I_{k m}$ for $k=1,2, \ldots$ and $m=2,3, \ldots$.

Proof. Under the assumptions of $(\mathrm{H}-0)-(\mathrm{H}-3)$, it is rather standard to verify that the Palais-Smale condition holds, see, e.g., [1, 14]. Therefore, the minimizers of $I_{k}$ and $I_{\infty}$ are achieved by some functions $u_{k} \in V_{k}$ and $u_{0} \in V_{\infty}$ for all $k$. 
Let $u(x, y)$ be a minimizer of $J(u)$ on $V_{k m}$. Since $I_{k m}<I_{\infty}$,

$$
\partial u / \partial x \not \equiv 0 \text { on } \Omega_{a} \text {. }
$$

Let

$$
v(x, y)= \begin{cases}u\left(\frac{x}{m}, y\right) & \text { for } x \in(-a, a) \text { when } k m \text { is odd } \\ u\left(\frac{x+a}{m}, y\right) & \text { for } x \in(-a, a) \text { when } k m \text { is even. }\end{cases}
$$

Then for $k m$ odd we have

$$
v\left(x+\frac{4 a}{k}, y\right)=u\left(\frac{x}{m}+\frac{4 a}{m k}, y\right)=u\left(\frac{x}{m}, y\right)=v(x, y) .
$$

Furthermore, we have

$$
\begin{aligned}
\int_{\Omega_{a}}|\nabla v|^{2} & =\int_{\omega} \int_{-a}^{a}\left\{\left(\frac{\partial v}{\partial x}\right)^{2}+\left(\nabla_{y} v\right)^{2}\right\} d x d y \\
& =m \int_{\omega} \int_{-a / m}^{a / m}\left\{\frac{1}{m^{2}}\left(\frac{\partial u}{\partial x}\right)^{2}+\left(\nabla_{y} u\right)^{2}\right\} d x d y<\int_{\Omega_{a}}|\nabla u|^{2} .
\end{aligned}
$$

Similarly, if $\mathrm{km}$ is even then (3.5) and (3.6) can also be proved. Since $u$ and $v$ are periodic in $x$ of periods $\mathrm{km}$ and $k$, respectively, it can be proved that

$$
\int_{\Omega_{a}} v f(v)=\int_{\Omega_{a}} u f(u) \text { and } \int_{\Omega_{a}} F(v)=\int_{\Omega_{a}} F(u) .
$$

Therefore, (3.6) and (3.7) imply $M(v)<0$. Since $f^{\prime}(0)=0$, we have $M(t v)>$ 0 for $t>0$ and sufficiently small. This implies that there exists a $t_{1} \in(0,1)$ such that $M\left(t_{1} v\right)=0$.

Now, by (H-2), we have

$$
\frac{d}{d t}\left\{\frac{1}{2} t u f(t u)-F(t u)\right\}=\frac{u}{2}\left\{t u f^{\prime}(t u)-f(t u)\right\}>0
$$

for $u>0$. Therefore, we have

$$
\begin{aligned}
I_{k} & \leq J\left(t_{1} v\right)=\int_{\Omega_{a}} \frac{1}{2} t_{1}^{2}|\nabla v|^{2}-F\left(t_{1} v\right) \\
& =\int_{\Omega_{a}} \frac{1}{2} t_{1} v f\left(t_{1} v\right)-F\left(t_{1} v\right)<\int_{\Omega_{a}} \frac{1}{2} v f(v)-F(v) \\
& =\int_{\Omega_{a}} \frac{1}{2} u f(u)-F(u)=I_{k m} .
\end{aligned}
$$

The proof is complete.

Proof of Theorem 1.1. For each $k \geq 1$ and $a \in\left(a_{k}, \infty\right), a_{k}$ given by (2.14), the minimizers of $I_{j}(a)$ and $I_{\infty}(a)$ are achieved by some functions $u_{j} \in V_{j}$ and $u_{0} \in V_{\infty}, j=1, \ldots, k$. By $(3.3), I_{j}(a)<I_{\infty}(a), j=1, \ldots, k$.

Now let $1 \leq i<j \leq k$ and $l$ be the least common multiple of $i$ and $j$. If $j=l$ then, by Lemma 3.2, $u_{i}$ and $u_{j}$ are different. If $j<l$ then $V_{i} \cap V_{j}=V_{l}$. If $u_{i}=u_{j}=\tilde{u} \in V_{l}$ then

$$
I_{l} \leq J(\tilde{u})=I_{i}<I_{\infty}
$$


By (3.8) and Lemma 3.2, we obtain $I_{i}(a)<I_{l}(a)$, a contradiction to (3.8). Hence, $u_{i}$ and $u_{j}$ are different. The proof is complete.

\section{SHORT CYLINDERS}

In this section, we shall prove that there is no asymmetric positive solution of (1.1)-(1.3) when the cylinders are short enough.

We need the following a priori estimates.

Lemma 4.1. Assume conditions (H-0)-(H-3)' are satisfied. Then there exists a constant $C=C(\omega)>0$ such that for any $a>0$ and any positive solution $u_{a}$ of (1.1)-(1.3), we have

$$
\left\|u_{a}\right\|_{\infty}=\max \left\{\left|u_{a}(x)\right|: x \in \Omega_{a}\right\} \leq C .
$$

Proof. Since the extension $\tilde{u}_{a}$ of $u_{a}$ is smooth in $\Omega_{\infty}$, i.e., $\tilde{u}_{a} \in C^{2}\left(\bar{\Omega}_{\infty}\right)$, the boundary $\{-a, a\} \times \omega$ is now an interior part of $\Omega_{\infty}$. Since $\tilde{u}_{a}>0$ in $\Omega_{\infty}$ and satisfies

$$
\begin{aligned}
\Delta \tilde{u}_{a}+f\left(\tilde{u}_{a}\right)=0 & \text { in } \Omega_{\infty}, \\
\tilde{u}_{a}=0 & \text { on } \partial \Omega_{\infty},
\end{aligned}
$$

by using the technique of "blow up" as in [7], we can prove (4.1). The details are omitted.

Proof of Theorem 1.2. For $a>0$, if $u_{a}$ is an asymmetric positive solution of (1.1)-(1.3), then

$$
w_{a} \equiv \partial u_{a} / \partial x \not \equiv 0
$$

and $w_{a}$ satisfies

$$
\begin{aligned}
\Delta w_{a}+f^{\prime}\left(u_{a}\right) w_{a}=0 & \text { in } \Omega_{a}, \\
w_{a}=0 & \text { on } \partial \Omega_{a} .
\end{aligned}
$$

Let $\tilde{\lambda}_{1}(a)>0$ be the least eigenvalue of the Laplacian $-\Delta$ on $\Omega_{a}$ with Dirichlet boundary condition. Then it is easy to verify that

$$
\lim _{a \rightarrow 0^{+}} \tilde{\lambda}_{1}(a)=\infty \text {. }
$$

On the other hand, the least eigenvalue $\eta_{1}(a)$ of the linearized eigenvalue problem

$$
\begin{aligned}
\Delta w+f^{\prime}\left(u_{a}\right) w & =-\eta w & & \text { in } \Omega_{a}, \\
w & =0 & & \text { on } \partial \Omega_{a}
\end{aligned}
$$

can be characterized by

$$
\eta_{1}(a)=\inf \left\{Q(v) / \int_{\Omega_{a}} v^{2}: v \in H_{0}^{1}\left(\Omega_{a}\right) \backslash\{0\}\right\},
$$

where

$$
Q(v)=\int_{\Omega_{a}}|\nabla v|^{2}-f^{\prime}\left(u_{a}\right) v^{2} .
$$

By Lemma 4.1, there exists a constant $C_{1}>0$ such that

$$
\left\|f^{\prime}\left(u_{a}\right)\right\|_{\infty} \leq C_{1} .
$$


By the Poincare inequality, we have

$$
\int_{\Omega_{a}}|\nabla v|^{2} \geq \tilde{\lambda}_{1}(a) \int_{\Omega_{a}} v^{2}
$$

for all $v \in H_{0}^{1}\left(\Omega_{a}\right)$. Hence, by (4.5), (4.6), and (4.7), there exists $a_{*}>0$ such that

$$
\eta_{1}(a)>0
$$

for all $a \in\left(0, a_{*}\right)$. Therefore, (4.2), (4.3), (4.4), and (4.8) imply that there is no asymmetric positive solution for $a \in\left(0, a_{*}\right)$. The proof is complete.

Remark 4.2. The results are still valid if $f(u)$ is replaced by $f(y, u)$, which satisfies similar conditions as (H-0) $-(\mathrm{H}-3)$ and $(\mathrm{H}-3)^{\prime}$.

\section{ACKNOWLEDGMENT}

The author is grateful for several useful conversations with Professor H. Berestycki during his stay in Taiwan in April 1988.

\section{REFERENCES}

1. A. Ambrosetti and P. Rabinowitz, Dual variational methods in critical point theory and applications, J. Funct. Anal. 14 (1973), 349-381.

2. H. Berestycki and F. Pacella, Symmetry properties for positive solutions of elliptic equations with mixed boundary conditions, preprint.

3. H. Brezis and L. Nirenberg, Positive solutions of nonlinear elliptic equations involving critical Sobolev exponents, Comm. Pure Appl. Math. 36 (1983), 437-477.

4. G. Cerami, Symmetry breaking for a class of semilinear elliptic problems, Nonlinear Anal. Theory, Methods, and Applications 10 (1986), 1-14.

5. C. V. Coffman, A nonlinear boundary value problem with many positive solutions, J. Differential Equations 54 (1984), 429-437.

6. E. N. Dancer, On non-radially symmetric bifurcation, J. London Math. Soc. 20 (1979), 287-292.

7. B. Gidas and J. Spruck, A priori bounds for positive solutions of nonlinear elliptic equations, Comm. Partial Differential Equations 6 (1981), 883-901.

8. Y. Y. Li, Existence of many positive solutions of semilinear elliptic equations on annulus, $\mathrm{J}$. Differential Equations 83 (1990), 348-367.

9. S. S. Lin, On non-radially symmetric bifurcation in the annulus, J. Differential Equations 80 (1989), 251-279.

10. __ Positive radial solutions and non-radial bifurcation for semilinear elliptic equations in annular domains, J. Differential Equations 86 (1990), 367-391.

11. __ Existence of positive nonradial solutions for elliptic equations in annular domains, Trans. Amer. Math. Soc. (to appear).

12. __ Symmetry breaking for semilinear elliptic equations on sectorial domains in $\mathbb{R}^{2}$, Proc. Roy. Soc. Edinburgh Sect. A 118 (1991), 327-353.

13. Z. Nehari, On a class of nonlinear second order differential equations, Trans. Amer. Math. Soc. 95 (1960), 101-123.

14. W.-M. Ni, Some aspects of semilinear elliptic equations, lecture notes, National Tsing Hua University, Hsinchu, Taiwan, May 1987.

15. M. Ramaswamy and P. N. Srikanth, Symmetry breaking for a class of semilinear elliptic problems, Trans. Amer. Math. Soc. 304 (1987), 839-845.

16. J. Smoller and A. Wasserman, Symmetry-breaking for solutions of semilinear elliptic equations, Arch. Rational Mech. Anal. 95 (1986), 217-225. 
17. __ Symmetry-breaking for solutions of semilinear elliptic equations with general boundary conditions, Comm. Math. Phys. 105 (1986), 415-441.

18. T. Suzuki and K. Nagasaki, Lifting of local subdifferentiations and elliptic boundary value problems on symmetry domains. I, Proc. Japan Acad. 64 (1988), 1-4.

19. _ On the nonlinear eigenvalue problem $\Delta u+\lambda e^{u}=0$, Trans. Amer. Math. Soc. 309 (1988), 591-608.

Department of Applied Mathematics, National Chiao Tung University, Hsin-chu, Taiwan, Republic of China 